Bundesgesundheitsbl - Gesundheitsforsch Gesundheitsschutz 2009 · 52:147-148 DOI 10.1007/s00103-009-0757-0

(c) Springer Medizin Verlag 2009

\section{G. Krause}

Robert Koch-Institut, Berlin, BRD iebe Leserin, lieber Leser,

eine zentrale Idee der Europäischen Union (EU) ist es, dass ihre Bewohnerinnen und Bewohner Anspruch auf gleichwertige Lebensbedingungen haben. Dazu vermag der Gesundheitsschutz sicher einen großen Beitrag zu leisten. Hier können vor allem Maßnahmen zum Schutz vor Infektionskrankheiten schnell grenzüberschreitende Dimensionen entwickeln. Es ist daher nicht überraschend, dass die Aktivitäten und Regelungen der EU, die den Informationsaustausch und die Koordination im Bereich des Infektionsschutzes zum Ziel haben, besonders vielfältig sind. Wie vielfältig, das veranschaulicht das hier vorliegende Schwerpunktheft.

In ihrem Beitrag über die EU-Strukturen zur Überwachung und Bekämpfung von Infektionskrankheiten beschreiben Schreck und Kollegen, wie komplex der Versuch ausfällt, weitreichende europaweite Abstimmungen bei bestehender Zuständigkeit der nationalen Ebenen zu ermöglichen. In der EU hat sich dabei eine beachtliche Entwicklung - von einer bloßen Projektförderung im Rahmen des „Europa gegen AIDS“-Programms im Jahr 1991 bis hin zur Gründung des Europäischen Zentrums zur Prävention und Kontrolle von Krankheiten (ECDC) in Stockholm im Jahr 2004 - vollzogen.

\title{
Infektionsschutz europäisch - von staatlicher Souveränität zu internationaler Vernetzung
}

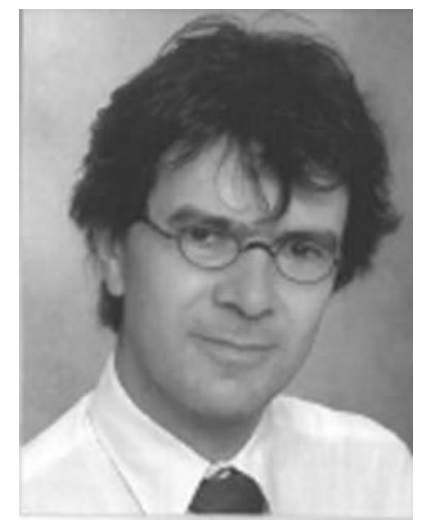

Im Bereich lebensmittelbedingter Zoonosen ist die europäische Integration weiter fortgeschritten, Informationsflüsse und Zuständigkeiten sind straffer und zentraler organisiert, wie die beiden Beiträge von Wichmann-Schauer und Ellerbrök sowie ihrer Koautoren zeigen. Mitunter verweisen Mitarbeiter der EU-Kommission im Bereich Public Health angesichts etablierter Labornetzwerke, weitreichender Informationspflichten seitens der Mitgliedstaaten und umfassender Interventionsmöglichkeiten der Kommission neidvoll auf ihre Kollegen aus dem Bereich Lebensmittelsicherheit. Doch dieser Vergleich lässt außer Betracht, dass es im Infektionsschutz vornehmlich um Menschen geht, um die Verbesserung der Gesundheit der Bevölkerung und nicht um tierische oder pflanzliche Produkte, die als Teil des gemeinsamen Marktes in ungleich höherem Maß Gegenstand von Integrationszielen und-maßnahmen sind. Der EG-Vertrag verpflichtet die EU dazu, die Verantwortung der Mitgliedstaaten für die Organisation des Gesundheitswesens und für die medizinische Versorgung in vollem Umfang zu wahren. Unbestritten ist dabei, dass es im Interesse aller Mitgliedstaaten ist, nationale Infektionsschutzkonzepte so zu gestalten, dass sie zumindest kompatibel mit denen benachbarter Staaten sind. Dies ist besonders augenfällig im Bereich der Influenzapandemieplanung, für den Haas und Kollegen in diesem Heft darlegen, inwieweit sich die nationalen Pandemiepläne der EU-Mitgliedstaaten ähneln bzw. voneinander unterscheiden. Gerade in der Pandemieplanung zeigt sich, wie die Mitgliedstaaten bei einem ausgesprochenen Wunsch nach Abstimmung doch auf ihre Eigenständigkeit beharren, was angesichts der betroffenen Zuständigkeitsbereiche und der enormen finanziellen Implikationen verständlich ist.

Gleichwohl, so scheint es, haben die Mitgliedstaaten bereits einen Teil ihrer Souveränität im Bereich des Infektionsschutzes zugunsten supranationaler Strukturen abgegeben - und dies bezeichnenderweise nicht nur an die EU, sondern deutlicher noch an die Weltgesundheitsorganisation (WHO). Hollmeyer und Kollegen erläutern die Melde- und Auskunftspflichten, die in den im Jahr 2007 in Kraft getretenen internationalen Gesundheitsvorschriften (IGV) formuliert sind. In Anlehnung an den Westfälischen Frieden von 1648 , in dem die Souveränität von Einzelstaaten partiell zugunsten eines Staatenbundes aufgegeben wurde, spricht Fidler 
bezüglich der IGV, die auch konkrete Standards zur Ausstattung des öffentlichen Gesundheitsdienstes (ÖGD) fordern, vom Eintritt in die postwestfälische Ära im Bereich Public Health (Fidler, Journal for Law and Medical Ethics, 2003). Lange vor den neuen IGV haben innerhalb der EU sogenannte Surveillancenetzwerke zu einem innereuropäischen Informationsaustausch und somit zur Früherkennung und Aufklärung international relevanter Epidemien beigetragen. Inzwischen bindet das ECDC diese Netzwerke schrittweise in ein umfassendes europäisches Surveillancesystem ein. Ammon und Faensen beschreiben die erreichten Fortschritte, aber auch die Herausforderung, Vergleiche innerhalb der EU bei methodisch kaum übereinstimmenden Datenquellen durchführen zu wollen. Damit kommen wir zurück zum Ursprung der Informationen, d. h. zum öffentlichen Gesundheitsdienst vor Ort, der die Daten nicht nur erhebt, sondern die eigentlichen Infektionsschutzmaßnahmen festzulegen und zu vollziehen hat. Wie die kaskadenartige Arbeitsteilung im Infektionsschutz zwischen den verschiedenen Ebenen eines föderalen Staates im Kontext der internationalen Verpflichtungen organisiert ist, erläutern Gottschalk und Kollegen in diesem Heft. Für diese Aufgabe werden infektiologisch und epidemiologisch hoch qualifizierte, international vernetzte Fachkräfte benötigt, um den angewandten In- fektionsschutz vor Ort, aber im internationalen Kontext umzusetzen. Genau an dieser Stelle setzen die von Bremer und Kollegen beschriebenen Ausbildungsprogramme für angewandte Epidemiologie an. Insbesondere das inzwischen vom ECDC betriebene European Programme for Intervention Epidemiology (EPIET) schafft hier den so oft geforderten europäischen Mehrwert, da es gemeinsam mit Ausbildungszentren in den Mitgliedstaaten internationale Experten ausbildet, die dem speziellen Anforderungsprofil genügen.

Das vorliegende Themenheft kann nur einige Aspekte des Infektionsschutzes in Europa hervorheben. Man mag angesichts der zahlreichen Strukturen, Regelungen und Organisationen im Bereich des europäischen Infektionsschutzes, der Gefahr interferierender Zuständigkeiten, dem intensiven Abstimmungsbedarf, der offenkundigen Komplexität und vermeintlichen Trägheit des Systems durchaus zweifeln, ob die EU bereits ausreichend für einen modernen grenzüberschreitenden Infektionsschutz gerüstet ist. Die jüngste Initiative der europäischen Kommission, das Regelwerk im Bereich des Infektionsschutzes zu vereinfachen und effizienter zu gestalten, ist jedenfalls nachdrücklich zu begrüßen. Bei dieser Überarbeitung müssen auch die Mitgliedstaaten darauf achten, dass dem Auftrag und den Belangen des nationalen, vor allem aber des örtlichen öffentlichen Gesundheits- dienstes Rechnung getragen wird. Seit Jahren ist nämlich zu beobachten, dass nationale und vor allem europäische PublicHealth-Institutionen gestärkt und ausgebaut werden, während die kommunalen, für den Infektionsschutz zuständigen Behörden einem spürbaren Ressourcenabbau ausgesetzt sind. Diese Entwicklung ist bedenklich. Kein noch so gut ausgestattetes Labornetzwerk, kein noch so lückenloses Surveillancesystem auf nationaler oder internationaler Ebene kann dafür sorgen, dass Kinder geimpft, Infektionsquellen beseitigt, Hygiene trainiert und mutmaßlich infektiöse Personen in Quarantäne genommen werden. Dazu wird qualifiziertes Personal mit moderner Ausstattung benötigt - nicht nur in Stockholm und anderen Metropolen Europas, sondern in jeder Gemeinde der Europäischen Union.

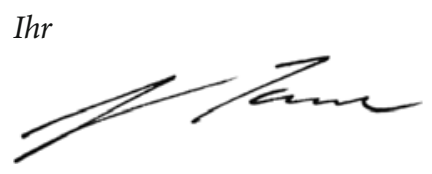

Gérard Krause

\section{Korrespondierender Autor}

\section{Dr. Gérard Krause}

Robert Koch-Institut

Postfach 650261

13302 Berlin, BRD

E-Mail: krauseg@rki.de 